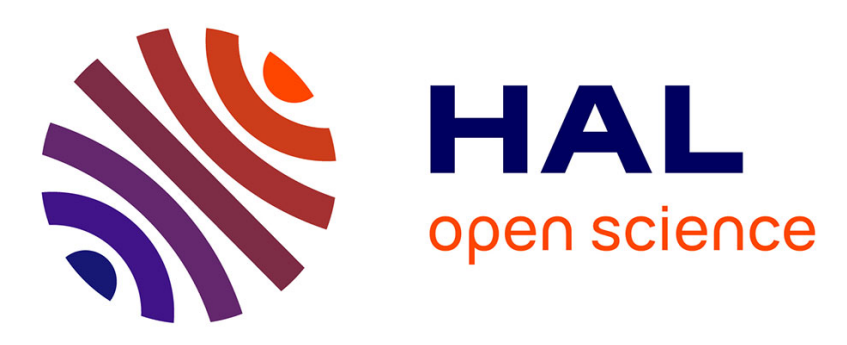

\title{
Crystal orientation mapping via ion channeling: An alternative to EBSD
}

Cyril Langlois, Thierry Douillard, Hui Yuan, Nicholas P. Blanchard, Armel

Descamps-Mandine, Bertrand van de Moortèle, Christophe Rigotti, Thierry

Epicier

\section{To cite this version:}

Cyril Langlois, Thierry Douillard, Hui Yuan, Nicholas P. Blanchard, Armel Descamps-Mandine, et al. Crystal orientation mapping via ion channeling: An alternative to EBSD. Ultramicroscopy, 2015, 157, pp.65-72. 10.1016/j.ultramic.2015.05.023 . hal-01226846

\section{HAL Id: hal-01226846 https://hal.science/hal-01226846}

Submitted on 21 Jun 2019

HAL is a multi-disciplinary open access archive for the deposit and dissemination of scientific research documents, whether they are published or not. The documents may come from teaching and research institutions in France or abroad, or from public or private research centers.
L'archive ouverte pluridisciplinaire HAL, est destinée au dépôt et à la diffusion de documents scientifiques de niveau recherche, publiés ou non, émanant des établissements d'enseignement et de recherche français ou étrangers, des laboratoires publics ou privés. 


\section{Crystal Orientation Mapping via ion channeling:}

\section{an alternative to EBSD}

C. Langlois $^{1 *}$, T. Douillard ${ }^{1}$, H. Yuan ${ }^{1}$, N.P. Blanchard ${ }^{2}$, A. Descamps-Mandine ${ }^{3}$, B. Van de Moortèle ${ }^{4}$, C. Rigotti $^{5}$, T. Epicier ${ }^{1}$

${ }^{1}$ University of Lyon - INSA de Lyon - CNRS, MATEIS, UMR 5510, Bât. Blaise Pascal, 20 Avenue Albert Einstein, 69621 Villeurbanne, France

${ }^{2}$ University of Lyon - CNRS, ILM, UMR 5306, Université Lyon I, Bât. A. Kastler, 10 rue A. Byron, 69622 Villeurbanne, France

${ }^{3}$ University of Lyon - CNRS, INL, UMR 5510, Bât. B. Pascal, INSA de Lyon/Université Lyon I, 69621 Villeurbanne, France

${ }^{4}$ Ecole Normale Supérieure de Lyon - CNRS, LGL, 46 allée d'Italie, 69364 Lyon, France ${ }^{5}$ University of Lyon - INSA de Lyon - CNRS, LIRIS, UMR 5205, INRIA, Bât. Blaise Pascal, 20 Avenue Albert Einstein, 69621 Villeurbanne, France

Keywords : electron backscattered diffraction ; orientation mapping ; ion imaging ; focused ion beam ; texture ; channeling

\section{Abstract}

A new method, which we name iCHORD (ion CHanneling ORientation Determination), is proposed to obtain orientation maps on polycrystals via ion channeling. The iChord method exploits the dependence between grain orientation and ion beam induced secondary electron image contrast. At each position of the region of interest, intensity profiles are obtained from a series of images acquired with different orientations with respect to the ion beam. The profiles are then compared to a database of theoretical profiles of known orientation. The Euler triplet associated to the most similar theoretical profile gives the orientation at that position. The proof-of-concept is obtained on a titanium nitride sample. The potentialities of $\mathrm{iCHORD}$ as an alternative to EBSD are then discussed.

\footnotetext{
* Corresponding Author
} 


\section{Introduction}

Electron backscatter diffraction (EBSD) is routinely employed as a characterization tool coupled with a scanning electron microscope (SEM) to obtain individual grain orientations, local texture, point-to-point orientation correlations, and phase identification and distributions on the surfaces of bulk polycrystals [1]. As commercial EBSD systems are now readily and widely available, the technique is no longer reserved to specialized research laboratories. Consequently EBSD has become a tool of choice for process development and quality control. With the goal of full automation in mind, efforts are being made to increase the speed of the technique [2] to obtain orientation maps. Several avenues are currently being explored, such as increasing the sensitivity and speed of the digital camera [ $\underline{3}]$ as proposed by the manufacturers, as well as improving the indexation algorithms for treating and indexing patterns [4]. In this context, any new technique that can ease the implementation of orientation mapping at an industrial scale would be welcome. The precision of the indexation is also the subject of intense research efforts. Indexing algorithms tend to be more and more precise, revisiting the classical Hough transform used to extract the diffraction lines from the Kikuchi patterns []ㅡ []. Other approaches are currently explored, using for instance the pattern matching technique, which consist in comparing directly the Kikuchi patterns to a database of simulated ones. This idea was proposed originally by Wright et al. in 1991 [7] and was recently implemented again by Chen et al. with the benefits of modern computational power [8]. The same technique has been used in Transmission Electron Microscopy to obtain orientation maps using precession diffraction $[\underline{9}, \underline{10}]$.

We propose in this paper a new technique for orientation mapping based on the well-known channeling contrast phenomenon [11] [12]. As the channeling contrast is emphasized in ionic images [13], the experiments were conducted in a FIB-SEM instrument to acquire ionic image series as well as comparative EBSD orientation maps. According to the channeling theory for ions and electrons, the intensity of induced secondary electrons (SE) at a given position on the surface of a 
polycrystalline sample will change if the orientation of the primary incident beam with respect to the sample surface is changed [14]. An intensity profile can then be drawn as a function of the sample orientation, from which information about the orientation of the crystalline lattice at this position can be retrieved. This idea has been suggested several times in the literature [14-16], but, to our knowledge, no comprehensive method currently exists that is capable of yielding full orientation maps on common polycrystalline samples, such as pure metals or metallic alloys. A relevant contribution was recently brought by Veligura et al. [16] but orientation maps were obtained only for particles deposited in epitaxy on a substrate, with only a single degree of freedom in rotation for the lattice orientation of the different grains.

This paper is divided into several sections. In Section 2, the approach we used to obtain ionic image series is outlined, with a comparison between a tilting image series and a rotation image series. Section 3 deals with the experimental details and Section 4 addresses the question of the different possible ways to recover the crystallographic orientation associated with an experimental intensity profile. The results and performances obtained with our method on a TiN sample are exposed in Section 5. The following discussion (Section 6) addresses several important points such as the spatial resolution, the time to obtain an orientation map and the applicability of this technique to non-cubic materials as well as multi-phased materials. We conclude this study in Section 7, with an outline of our key results.

\section{Intensity profiles as a signature of the orientation}

As mentioned in the introduction, the channeling effect has been theoretically modeled both for ions and electrons [17]. These models include a significant role of lattice plane orientation with respect to the charged particle beam and channeling contrast has frequently been applied to enhance microstructural details, especially in SEM [수 $[\underline{19}][\underline{13}][\underline{15}, \underline{16}][\underline{20}]$. The work of Yahiro et al. clearly illustrates the variation of the SE signal under a $\mathrm{Ga}^{+}$probe, with minima appearing when the beam arrives parallel to low index crystallographic planes [14]. However, if the aim of an experiment is to recover the crystallographic orientation of the sample, acquiring a tilt series of images is clearly not 
the best choice for symmetry reasons. Usually, the tilt axis lies in the sample surface, let say along the $\mathbf{e}_{\mathbf{x}}$ direction as represented in the drawing of Figure 1 a and $b$ which represent respectively the reference frame, common to the sample and the stage, as well as its position in a stereographic projection. Within a tilt series, the ion or electron beam originally perpendicular to the sample surface along $\mathbf{e}_{\mathbf{z}}$ direction crosses the stereographic projection linked to the reference frame along a plane passing through the center of the projection, containing $\mathbf{e}_{\mathbf{y}}$ and $\mathbf{e}_{\mathbf{z}}$ directions. If we assume that the signal received only depends on the intersections between the beam and the crystallographic planes or directions, the same signal evolution will be obtained for two different crystals with orientations linked by mirror symmetry with respect to the geometric plane in which the beam travels. An example is given in Fig. 1c, showing two stereographic projections corresponding to such mirror orientations, with the beam path represented by a vertical dashed line. A beam tilted around the $\mathbf{e}_{\mathbf{x}}$ direction will cross the same planes at exactly the same angular positions on the two stereographic projections, giving the same intensity variations. This kind of situation does not allow an unambiguous determination of the crystallographic orientation from the obtained intensity profile and additional experiments would then be required. On the contrary, if we give a fixed tilt to the sample, for arguments sake $40^{\circ}$, and then rotate the sample around its tilted normal ( $\mathbf{e}_{\mathbf{z}}$ direction), the beam will circulate along a cone figured by the dotted circle in the stereographic projections of a given grain (see Figure 1c). A series of image acquired during such a rotation of the sample (around a rotation axis not parallel to the beam) then results in unique intensity profiles for a given orientation because there is no other stereographic projection that would result in intersections with the same planes at the same angular positions, with the same sense of rotation. In this work, we used rotation image series to obtain the orientation of the crystal for each point of the region of interest (ROI). Concerning the initial tilt, because of the necessity to cross enough planes to obtain a significant signature, we have chosen rather arbitrarily $40^{\circ}$. It is worth noting that a correction of beam deflection during the image scan has to be included if working at low magnifications [17]. In the present work, the magnification is $500 \mathrm{X}$, with an horizontal field of view of $230 \mu \mathrm{m}$. In these conditions, the beam deflection is low enough to ignore the effect. 
a)

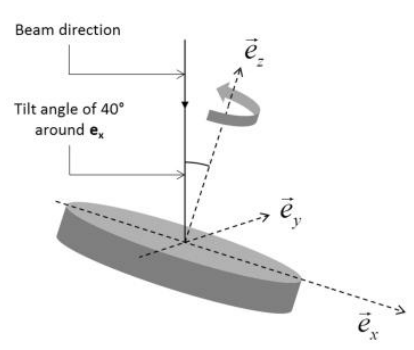

c)

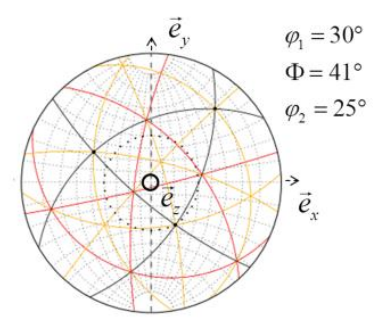

b)
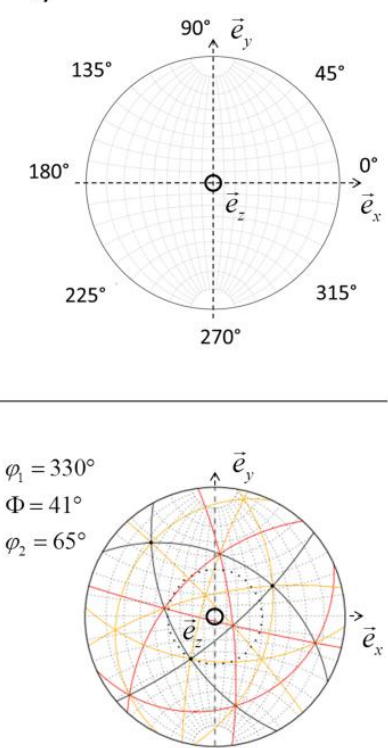

Figure 1 (color online): (a) schematic of the sample together with its orthonormal reference frame ( $\boldsymbol{e}_{\boldsymbol{x}}$ $\left.; \boldsymbol{e}_{y} ; \boldsymbol{e}_{z}\right)$ attached to the surface. The beam direction is represented vertically and the tilt angle is defined as the angle between the beam direction and the direction $\boldsymbol{e}_{\mathrm{z}}$ normal to the surface of the sample. (b) Stereographic projection attached to the sample with the representation of its reference frame. (c) stereographic projections attached to the sample, with the crystallographic plane families $\{100\}\{110\}$ and $\{111\}$ represented respectively in black, yellow and red (color online) for the two orientations Euler triplets indicated. The path followed by the beam during a tilt series around the $\boldsymbol{e}_{\boldsymbol{x}}$ axis is represented as a dashed line. The two crystallographic orientations represented are linked by a mirror symmetry with respect to the geometric plane $\left(\boldsymbol{e}_{\mathbf{y}} ; \boldsymbol{e}_{\mathrm{z}}\right)$ in which the beam travels. Note: the dotted circles on the two stereographic projections correspond to the beam path during a rotation series around $\boldsymbol{e}_{\mathbf{z}}$ assuming a fixed tilt of $40^{\circ}$.

\section{Experimental setup}

Experiments were carried out on a ZEISS NVision40 FIB-SEM machine in order to obtain both ioninduced electron images and reference orientation maps, acquired with an Oxford Instruments $\mathrm{F}^{\mathrm{TM}}$ EBSD system (fast camera Nordlysll and Channel 5 software). Concerning the ionic source, the microscope is equipped with a Seiko Zeta column and a Ga liquid metal ion source. FIB images were 
acquired at $30 \mathrm{kV}$ and a current of $3 \mathrm{nA}$. This quite high current was chosen to have a probe size of $170 \mathrm{~nm}$ that matched the pixel size in the images. Matching the probe size and the image pixel size allows milling the surface evenly, without localized craters during the acquisition of an image series [21]. Moreover, the dwell time per pixel was $0.1 \mu \mathrm{s}$, which limits milling, amorphisation and Ga implantation. With these conditions, the ion channeling does not fade out, even after completing the whole series of 360 images. A titanium nitride (TiN) sample was used to acquire ion image series and EBSD maps. Titanium nitride has a face-centered cubic structure with a TiN motif localized at the origin of the crystallographic cell, and in the middle of each face. The sample does not present any strain or any precipitate in its microstructure. The surface of the sample was prepared as for classical EBSD: mechanical polishing, and vibratory polishing using colloidal silica as the final preparation step.

\section{Computational approach}

4.1. Acquisition and processing of image stacks

The raw images acquired in the experimental conditions exposed in Section 3 were numerically treated within the Fiji software [22] in order to get an aligned stack of images. Firstly, all the images being acquired with a tilt of $40^{\circ}$, they are compressed in the direction perpendicular to the tilt axis and have to be rescaled. Then, as we chose to make a rotation series, each image must be corrected for its own rotation angle. Finally, residual drifts in X-Y directions were corrected [23] and the largest common area in all the images of the series was finally cropped. The final result is a stack of images reflecting the same area, with identical dimensions, but showing variations of contrast in each pixel due to the channeling phenomenon. At any position $(x, y)$ in the image, we obtain an intensity profile by representing the intensity variation along the depth of the stack, the number of the slice being linked to the rotation angle. Figure 2 illustrates the processing from aligned images to intensity profiles. 


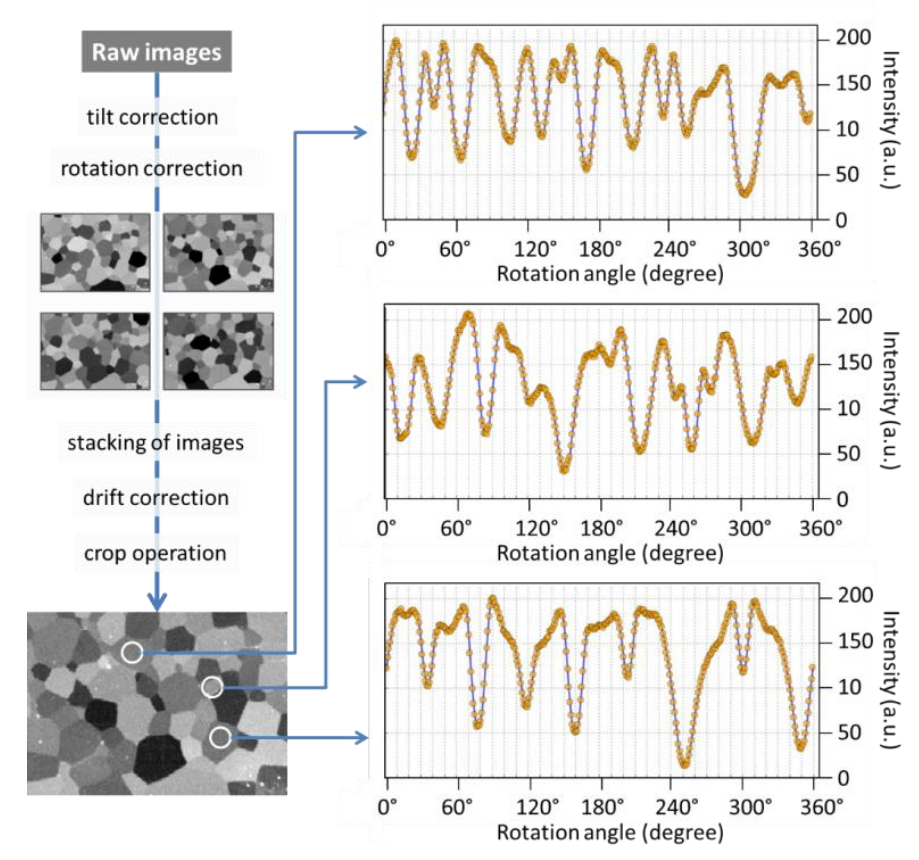

Figure 2 (color online): Diagram representing the successive process operations from the raw images to a fully aligned image stack from which intensity profiles can be extracted. Three representative intensity profiles are represented on the right side, coming from three different grains.

4.2. Linking intensity profiles and crystallographic orientation

Each intensity profile that can be extracted on each pixel of the ROI is a signature of the crystallographic orientation at that position. Several approaches are possible to recover the orientation from the intensity profiles.

The first one would be to analyze a given profile and to extract the significant information, like the plane family associated to each peak, as well as the angular separation between the peaks. Knowing the experimental tilt of the sample (here $40^{\circ}$ ) during the acquisition, this could be enough to determine geometrically the entire stereographic projection, and hence to come back to the three Euler angles. However, we observed that the peaks sometime correspond to the contribution of several planes, when the beam comes parallel or near a zone axis. Therefore, the value of an intensity minimum cannot be exclusively assigned to a given plane crossed by the beam. The second approach is a "brute force" technique which consists in constituting a theoretical database of the profiles corresponding to all the possible orientations for the material. Then, a search is carried out in 
the database to find the closest theoretical profile as compared to the experimental one, according to a given metric. Since the Euler angle triplet of this theoretical profile is known by construction, the orientation will be determined. This approach has been successfully used for determining orientations in a TEM by matching experimental precession diffraction patterns to a database of theoretical patterns $[\underline{9}, \underline{10}]$. In the present case, the key point is to construct the theoretical database.

This theoretical database must contain the intensity profiles associated to orientations represented by Euler triplets. To construct an intensity profile for a given orientation, one needs to know i) all the planes that will be crossed or closely approached by the beam ii) the shape of the peaks, iii) the loss amplitude attributed for each crystallographic plane, and iv) an adjustable factor to scale the calculated profiles to the intensity range of the experimental images. In our experimental setup, the planes giving a contribution in the profile are simply the ones that have a normal direction elevated by an angle less than $\left(90^{\circ}-40^{\circ}-\varepsilon\right)$ with respect to the surface of the sample. Here, the parameter $\varepsilon$ is a critical angle describing the limit out of which the beam does not "see" the plane. Normally, each family of planes has its own critical angle [17], but for simplicity's sake, the same $\varepsilon$ was taken for all the planes. A Gaussian curve was used to model the intensity loss observed when the beam crosses a crystallographic plane perpendicularly. Therefore, for each position of the beam (i.e. each abscissa position in the intensity profile) the angles between the beam and the planes considered are calculated, and the contributions of each plane to the intensity loss are added. It is worth noting that, with this method, when the beam crosses a plane perpendicularly, the loss peak is quite narrow whereas when the beam crosses a plane at a grazing incidence, the peak is wider. Moreover, due to this "critical angle", even if the beam does not geometrically cross a plane, it can be close enough to the plane (angle to the plane between 0 and $\varepsilon$ ) to give a significant channeling. By trial and error using the Graphical User Interface presented in Figure 3, the value for the Gaussian curve full width at half max has been fixed to $7^{\circ}$. Then, the intensity loss associated with each plane (i.e the depth of the Gaussian curve associated to a plane) must be determined. In this feasibility experiment, we can measure this parameter accurately by acquiring an EBSD map on the same area 
to construct the stereographic projection associated to a given experimental profile obtained in a given position. Knowing both the stereographic projection and the experimental profile, one can index the peaks in the profile and determine which intensity loss is associated to which family of lowindex planes. Figure 3 presents the Graphical User Interface (GUI) that has been coded to help determine the intensity loss assigned to each crystallographic plane from the EBSD data and experimental profiles. The values of intensity losses for the three main plane families are, in arbitrary units: 1000 for $\{100\}, 550$ for $\{110\}$ and 358 for $\{111\}$ (arbitrary units). A global scale factor has been fixed to 10 to account for the fact that the experimental data have not been normalized. It was found that considering only the $\{100\},\{110\}$ and $\{111\}$ planes families was enough to obtain a good fit to the experimental profiles.

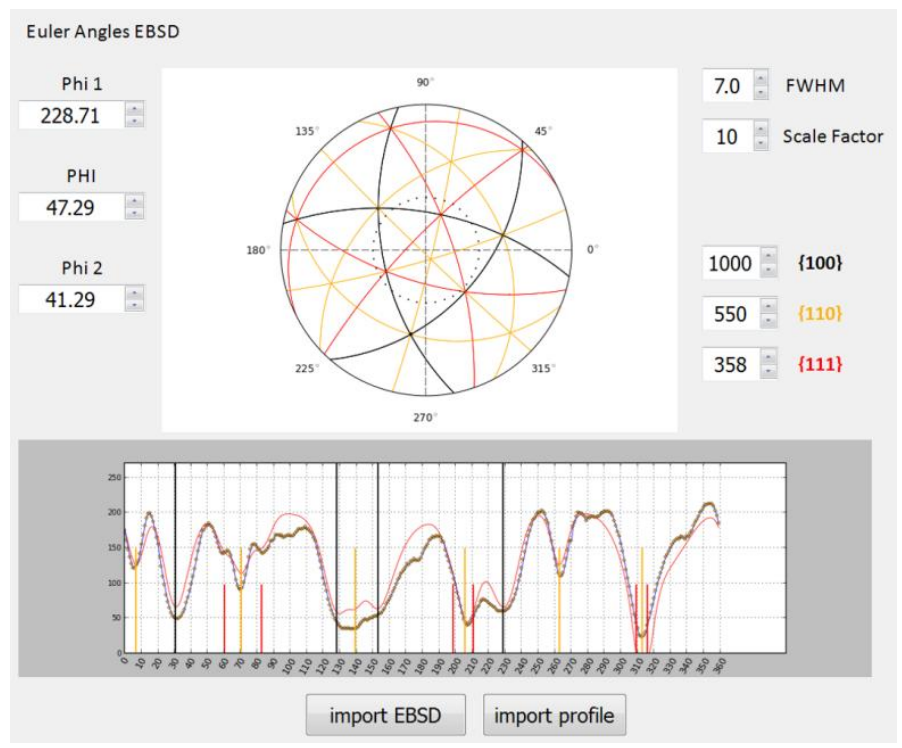

Figure 3 (color online): Graphical User Interface displaying the crystallographic orientation (the three Euler angle on the left, from EBSD experiment) and the corresponding intensity profile for a given position (orange dots on the bottom graph, from the rotation image series). On the intensity profile, the vertical lines represent the angular positions of the different crystallographic planes crossed by the beam during the rotation series (dotted circle on the stereographic projection). The color code is the following: black for $\{100\}$ plane family, yellow for $\{110\}$ plane family and red for $\{111\}$ plane 
family. The rotation angle starts from the $0^{\circ}$ position on the right side of the dotted circle and the sense of rotation along the dotted circle is anti-clockwise. The theoretical profile is represented in red on the graph. The controls on the right allow the user to modify visually the parameters of the theoretical profile to obtain the best fit to the experimental profile. See part 4.2 of the article for the meaning and values of these parameters.

All theoretical profiles can be stored in a database together with their corresponding Euler triplets. The link between an experimental profile, acquired during the sample rotation, and the crystallographic orientation can be established by searching in this database to find the theoretical profile that is the most similar to the experimental one and to retrieve the associated Euler triplet. As for the acquisition, performance is an issue since the database that has to be generated can be very large, especially if the step used for each Euler angle is small, and because in addition the search through the database has to be repeated for each position in the area of interest. Fast selection and access to stored data have been intensively investigated for several decades in the database research field. For instance, data structures as B trees and B+ trees [24] have been designed to offer sub-linear access costs with respect to the size of the database. This means that, when using these kinds of data organization, if the size of a database is multiplied by $\mathrm{K}$, then the time needed to retrieve an object according to a simple selection criterion will also increase, but less than by a factor of $\mathrm{K}$.

In the context of intensity profiles, each object is a profile described as a sequence of $\mathrm{N}$ intensity values ( $\mathrm{N}$ being the number of images in the image series) and the selection process is to find among a set of theoretical profiles the one that is the most similar to a given experimental profile. Each profile is a point in a space having $\mathrm{N}$ dimensions, and the problem can be reformulated as the search for the theoretical profile that is the closest to the experimental profile using a distance in this $\mathrm{N}$ dimensional space. So, all theoretical profiles are stored in a R-tree [도], that is a data structure designed to support the fast retrieval of multi-dimensional information from databases. Informally, an R-tree is a tree representing a decomposition of the data space in hyper-rectangles that are 
bounding boxes of sets of points. At the root of the tree, is a hyper-rectangle $\mathrm{H}$ that encompasses the whole set, S, of the points stored in the database. Each child of the root is a node storing a hyperrectangle included in $\mathrm{H}$, that is a bounding box of a subset of $\mathrm{S}$. This refinement is repeated along the branches, and the points are stored at the leaf level. This structure is used to find efficiently the nearest neighbor of a given point in space [26], with the Euclidean distance as metric.

To generate the theoretical profile database, the Euler angle steps were initially chosen as $3^{\circ}, 2^{\circ}$ and $2^{\circ}$ respectively for $\varphi_{1}, \Phi$ and $\varphi_{2}$. Owing to the cubic symmetry of the titanium nitride TiN lattice, the Euler angle $\varphi_{1}$ varies from $0^{\circ}$ to $360^{\circ}$, and $\Phi$ and $\varphi_{2}$ vary from $0^{\circ}$ to $90^{\circ}$ [27]. With this mapping of the Euler space, there is a maximum disorientation of 2.7 degrees between adjacent orientations in the database. This results in a database of 243,000 theoretical profiles.

To execute the database searches, the method takes direct advantage of the presence of several processor cores by distributing the set of experimental profiles over the cores, each process working with its own copy of the R-tree (containing the theoretical profiles). Since no communication is required between the processes, the execution time still scales linearly with respect to the number of experimental profiles, as if a single core were used. This is confirmed by Figure 4a that reports the whole search execution time using 30 cores when changing the number of experimental profiles to process (all profiles described in a space having 90 dimensions, simulating a case where the image series contains 90 images). The benefits of using a R-tree are underlined Figure $4 \mathrm{~b}$ by the curve depicting the search time when varying the size of the database. This figure shows that the search time scales nicely in a sub-linear way with respect to the size of the theoretical profile databases (execution times reported for a single core, a set of 80 experimental profiles to process, using 90 dimensions per profile) 

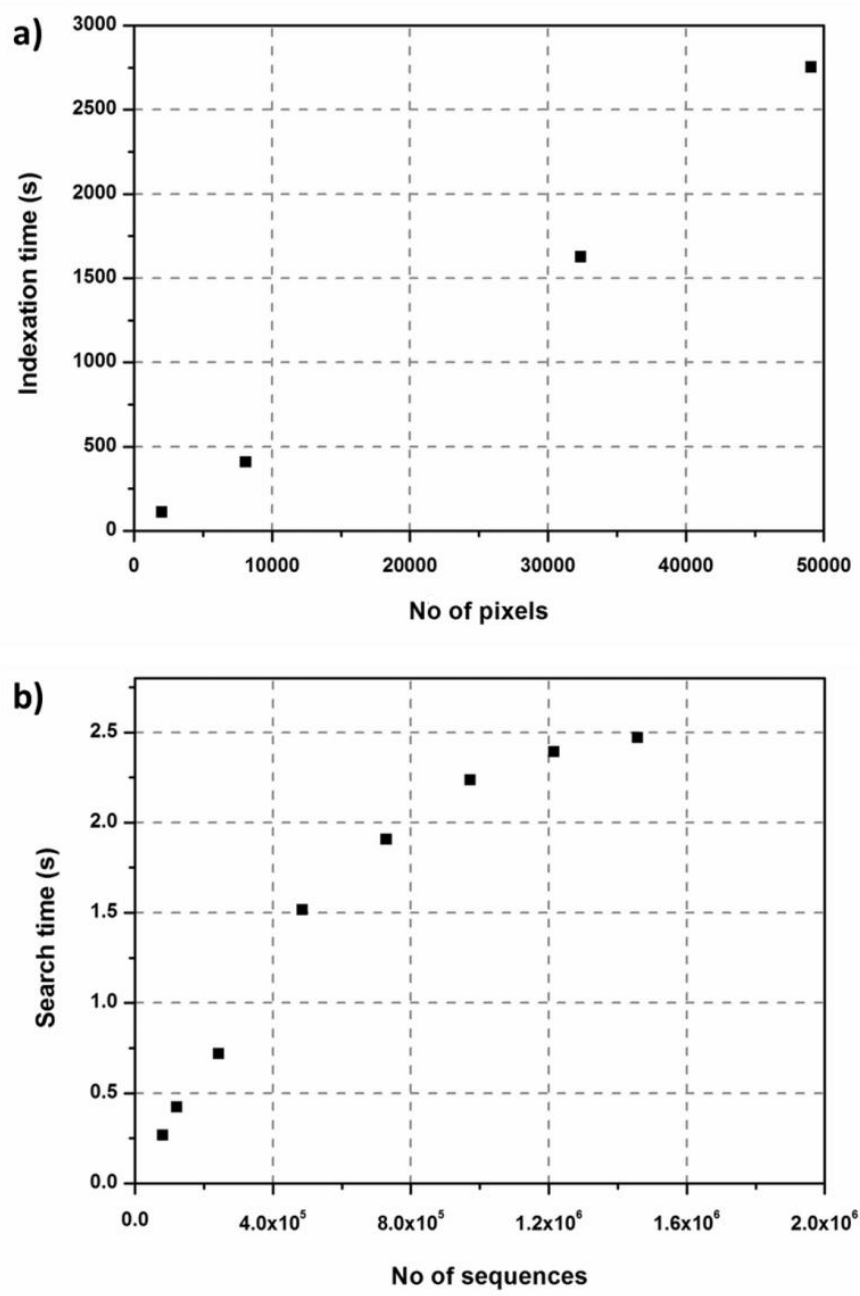

Figure 4: (a) graph showing the indexing time (seconds) of images of different sizes (in pixels). The experimental profiles used to search in the database are subsets of 90 values instead of 360, extracted from original profiles - one out of four, keeping the full range of $360^{\circ}$ - as described in section 5.2. (b) graph showing the search time in databases of different resolution in the Euler space (i.e. different sizes of database as represented along the abscissa).

\section{Results and performances}

5.1. Full database search

We used the EBSD map acquired on the same area as a reference to evaluate the correctness of the iChord indexation. In the context of a 'proof-of-concept', we evaluate that a disorientation lower than $5^{\circ}$ between the iChord orientation and the EBSD orientation at the same position can be considered as acceptable. To compare the results of the iChord method and the EBSD results 
(considered as a reference), the iChord and the EBSD data have been treated to have a common color code representing the Euler angles. The treatment, for a given orientation from EBSD or iChord maps, consist in choosing among the three symmetry-equivalent Euler triplets present in the Euler space (phi $10^{\circ}-360^{\circ}$; $\mathrm{PHI} 0^{\circ}-90^{\circ}$; phi2 $0^{\circ}-90^{\circ}$ ) the one with the largest phi1 value [28]. These three equivalent triplets in the Euler space result from a rotation about the three-fold axis of the cubic sample. Then, the color code used in Figure $5 a$ and $5 b$ is a proportional correspondence between the three Euler angles and values from 0 to 255 respectively for the three Red, Green and Blue (RGB) channels of the 8 bit color images. This allows comparing visually the iChord and EBSD orientations. For a quantitative comparison, the disorientation between homologous pixels in the iChord and the EBSD maps must be computed. To have a pixel per pixel correspondence between the iChord and the EBSD maps, it is necessary to apply an affine transform to the EBSD to correct from spatial distorsions between the two images. After this affine transformation, a position on the sample surface is represented by the same $(X, Y)$ coordinates both in the iChord map and the EBSD map. It is worth noting that this affine transformation does not alter the values of the Euler angles stored in the EBSD maps. The iChord map is presented on Figure 5a, the EBSD map after affine transformation is presented on Figure 5b, and the disorientation map is presented on Figure 5c. On Figure 5c, dark blue corresponds to $0^{\circ}$ of disorientation, and white corresponds to a disorientation of $5^{\circ}$ or higher. 
a)

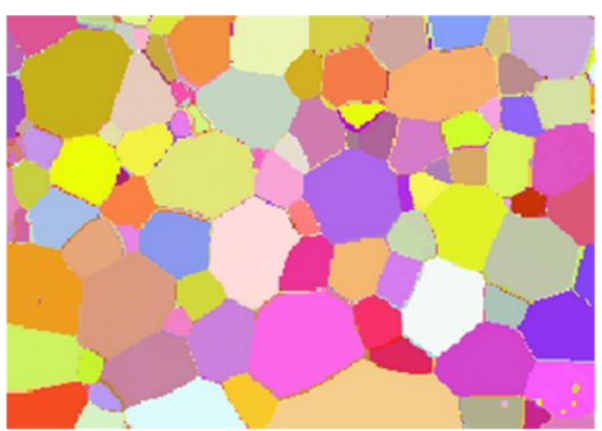

b)

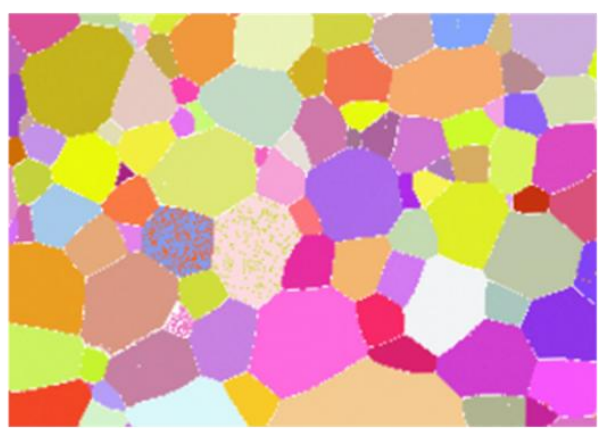

\section{$20 \mu \mathrm{m}$}

c)

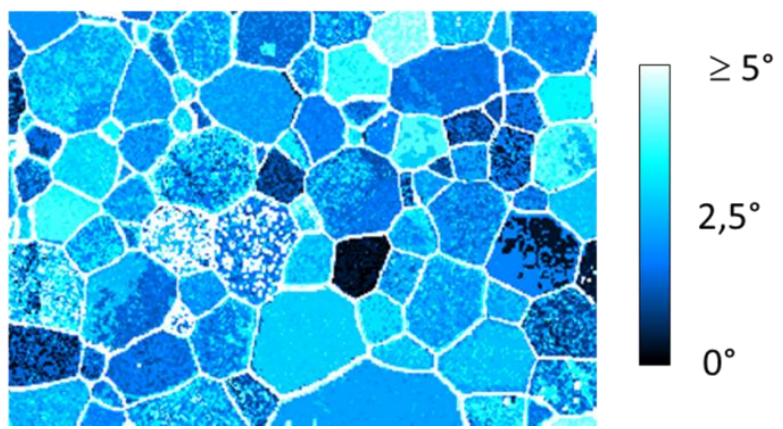

Figure 5 (color online): (a) Orientation map obtained with the iCHORD method presented in this work.

(b) Orientation map on the same area as (a) obtained from an EBSD experiment. (c) Disorientation map between (a) and (b) maps, with the following color code : dark blue corresponds to $0^{\circ}$ disorientation, and white corresponds to $5^{\circ}$ of disorientation or higher. The max disorientation found among the grains is $4.8^{\circ}$. Note: orientation maps have been treated to display Euler angles with the same color code (see part 5.1 of the article). An affine transform has been applied to the EBSD map to correct for the spatial distortions between the ion images and the EBSD image scan.

It is clear from Figure $5 c$ that all the grains have been indexed with a disorientation under $5^{\circ}$ compared to EBSD results. The mean disorientation over all the grains is $3.1^{\circ}$. We can conclude that, globally, our method allows the crystallographic orientation of large numbers of grains to be determined via a series of images showing channeling contrast. 


\subsection{Decreasing the acquisition time}

A potential application of this method could be to rapidly obtain information about the texture of a material. To minimize the acquisition time, the rotation series should consist of the least possible number of images. The number of images can easily be reduced, for example from 360 to 90 in two different manners: (i) a rotation step of $2^{\circ}$ and a rotation range of $180^{\circ}$, or (ii) a rotation step of $4^{\circ}$ and a rotation range of $360^{\circ}$. The results of these two "virtual" experiments (images were simply extracted from the same set of images as previously) are presented in Figure 6. Clearly, restricting the rotation range reduces the accuracy of the orientation map, with more incorrect indexations (Figure 6a). On the contrary, taking a rotation step of $4^{\circ}$ but keeping the full rotation range of $360^{\circ}$ gives a disorientation map (Figure $6 \mathrm{~b}$ ) identical to the one obtained by using the full set of images.

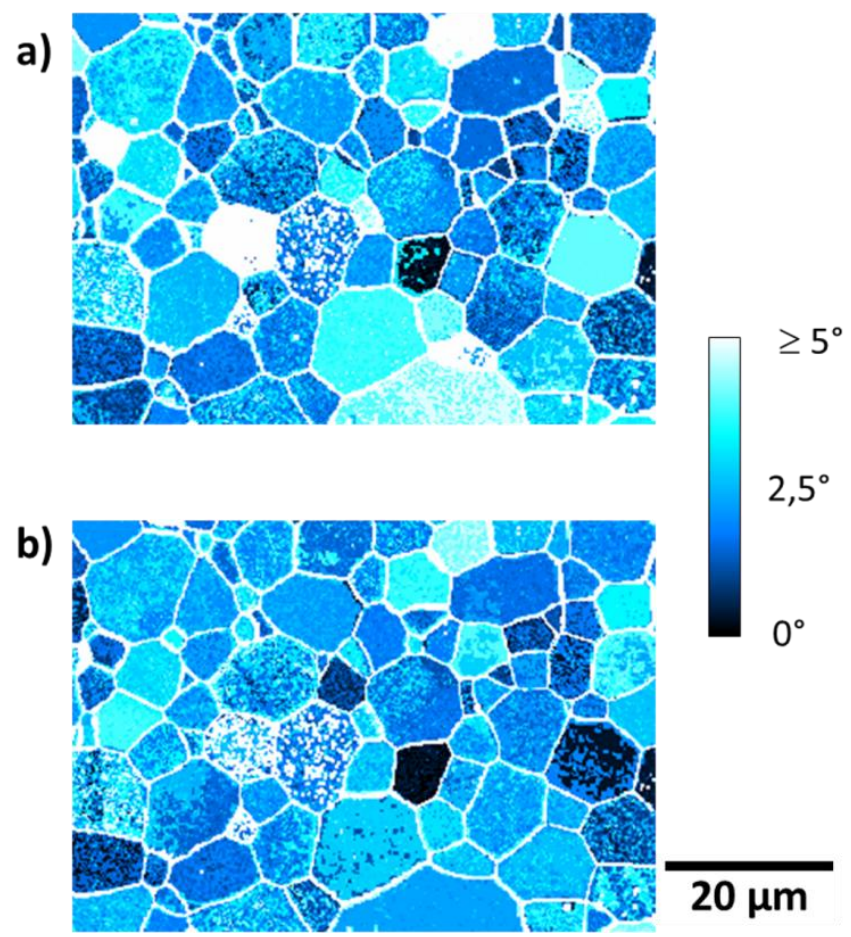

Figure 6 (color online): (a) disorientation between the averaged EBSD map and an iCHORD map using a reduction of dimension of (step $2^{\circ}$ : range $\left.180^{\circ}\right)$ - 90 images used, see section 5.2. (b) disorientation between the averaged EBSD map and an iCHORD map using a reduction of dimension of (step $4^{\circ}$ : 
range $\left.360^{\circ}\right)$ - 90 images used, see section 5.2. The max disorientation found among the grains in (b) is $4.8^{\circ}$.

One can wonder how far we can decrease the number of images while still preserving a good final accuracy of the orientation map. As a second attempt, we tried a setting of (rotation step : rotation range) equal to $\left(12^{\circ}: 360^{\circ}\right)$, which means only 30 images were needed. With these conditions, the orientation map obtained, presented on Figure 7, exhibits a slightly higher proportion of incorrect indexation compared to the $\left(1^{\circ}: 360^{\circ}\right)$ map. A full statistical study would be necessary to evaluate the effect of this degradation on the texture characterization. In the present proof-of concept experiment, we did not perform this analysis simply because it will remain a case-by-case study without any universal tendency.

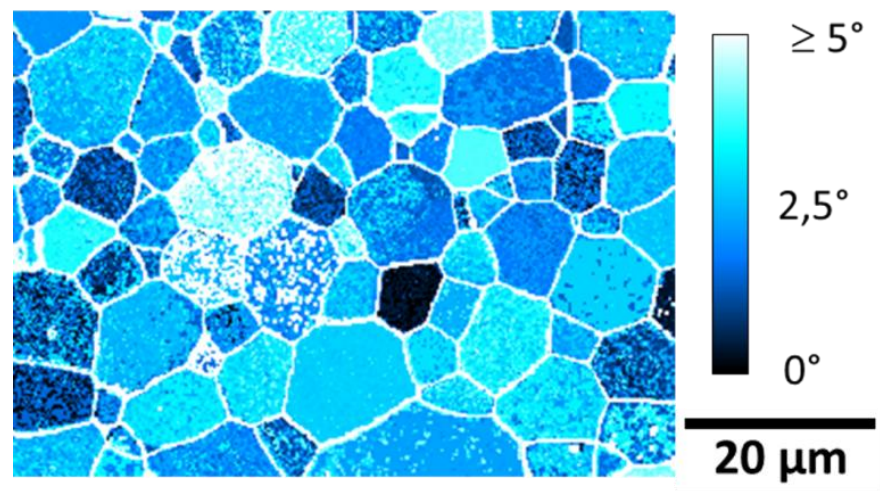

Figure 7 (color online): disorientation between the averaged EBSD map and an iCHORD map. Dark blue corresponds to $0^{\circ}$ disorientation and white corresponds to a disorientation of $5^{\circ}$ or higher. Grain boundaries have not been taken into account in this map. The dimensional reduction in this case was $\left(\right.$ step $12^{\circ}$ : range $\left.360^{\circ}\right)$ - see section 5.2., which means that only 30 images were needed to obtain this disorientation map.

\section{Discussion}

We have proposed a new method based on channeling contrast in order to measure the crystallographic orientation of polycrystals by acquiring a series of images from a tilted sample in a FIB-SEM. Using a model sample, it has been proven that the same information as the EBSD technique 
can be obtained. It means that all the data treatments usually made after an EBSD experiment in order to characterize the statistical distribution of grain orientation (pole figure, texture, etc.) can be attained with this method. The main difference with respect to EBSD is the way to obtain the Euler angle triplet (phi1, PHI, phi2) on every point of a ROI. Moreover, the acquisition and processing time are potentially small, with only a few tens of images being necessary and a very small processing time (once optimized) due to its nature - a search by similarity in a database. If the time to obtain texture information is the key-point, there is also another way to speed up the processing, by considering a mean orientation for each grain. From the image series, it is in principle easy to obtain the contour of each grain by an edge detection and averaging z-projections over the whole image stack, and consequently to deduce the positions of each grain center. Interestingly, whereas this operation is hazardous when using a single 2D image (generally because of a poor signal-to-noise ratio and/or the lack of contrast between some adjacent grains), it is quite straightforward when considering the rotation stack of images as acquired in the present method. Figure 8 presents an example for the present case with edge detection on single images and a summation of such images over the whole stack. Figure $8 a, b, c$ and $d$ show respectively: the original image stack (first image represented), an example of edge detection on a single image of the stack, the sum of all edge-detected images of the stack (360 images) and the sum of only 10 edge-detected images from the stack. Some details become hardly visible when comparing the sum of 360 edge-detected images and the sum of only 10 edge detected images. Summing only ten images is the lowest limit to be able to detect clearly all the grain boundaries. 
a)

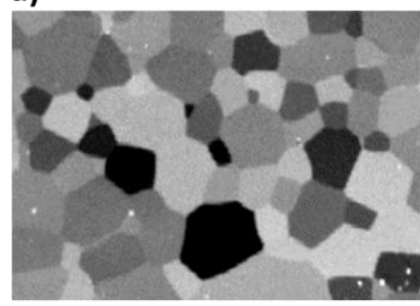

c)

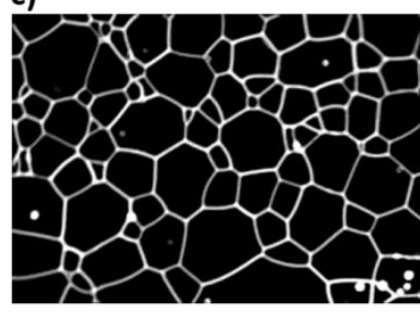

b)

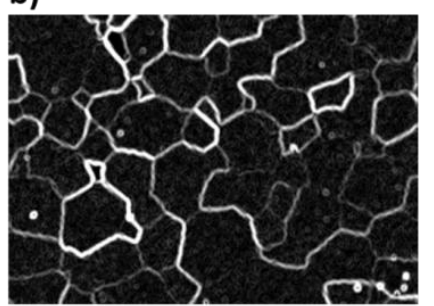

d)

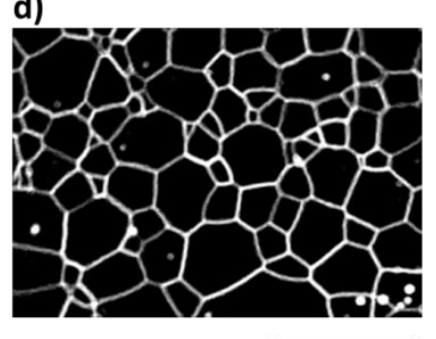

$20 \mu \mathrm{m}$

Figure 8: (a) first image of the rotation image series. (b) edge detection on a single image of the stack, representative of other edge-detected images computed from the stack. (c) sum of all the 360 edge-detected images computed from the rotation image stack. (d) sum of 10 edge-detected images extracted every 36 images from the rotation image stack.

A mean indexation for each grain can then be determined by indexing a few points around its center. In our case, the ROI contains a few hundred grains, which means that only a few hundred indexing operations are necessary, instead of indexing all pixels of the image $(264 \times 186=49104$ pixels). This approach would not be possible with classical EBSD because a complete scan has to be done first before knowing the contours of all the grains. However, some interesting solutions have been proposed to determine the position of the grain boundaries using the forward-scatter detectors

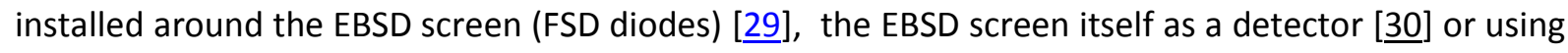
a scanning mesh refinement technique $[\underline{2}, \underline{31}]$.

Another improvement brought by this new method, as compared to EBSD, might be the lateral spatial resolution of the orientation map. The small depth of penetration of the ion beam as well as the geometry of acquisition are favorable concerning the spatial resolution. On the contrary, a drawback of using a relatively high atomic number ion beam such as $G$ a, is surface sputtering as well as possible ion implantation and amorphisation effects. In this case, using an $\mathrm{He}+$ beam would be a 
benefit [16]. However by correctly choosing the ion beam conditions (energy, dose, beam overlap), these effects will be negligible during the acquisition time of the azimuthal series.

Another important aspect is the possibility to implement this method as a stand-alone technique. As mentioned in section 4.2, we used an EBSD map of the same ROI to determine the intensity loss associated to each plane. This does not mean that an EBSD pre-calibration will be systematically required when applying the method to any new material. Intuitively, one may suggest that the intensity drop off should be directly linked to the scattering power of the corresponding plane family. Indeed, it was easy to check afterwards that using coefficients, for each plane, proportional to their structure factor allows pertinent intensity profiles to be obtained. This implies that theoretical or more appropriately simulated profiles could thus be constructed without the help of an EBSD map. It further means that two materials with the same symmetry (i.e., cubic as tested so far) but with different motif and Bravais lattice could be differentiated.

To totally evaluate the general interest of this new method, it will be necessary to further investigate (i) the spatial resolution of the technique, (ii) the accuracy of the indexation, (iii) if it can be applied to non-cubic materials, (iv) if it allows distinguishing between two (or more) crystallographic phases in the sample, (v) if it can be applied even if the channeling contrast is not very intense (for instance for heavily deformed materials). These goals are not in the scope of the present investigation, but will be the subject of further work.

A final question remains concerning the applicability of this technique to the SEM. Although the availability of FIB instruments is increasing, SEM instruments remain by far more largely installed in most laboratories and industries dealing with materials, in research and development or quality / control units. The possibility to obtain orientation maps only by imaging, without supplementary hardware would then be much welcomed. Among the challenges to take up with electrons, one has to consider the smaller intensity of the channeling contrast, the larger depth of penetration in the sample, or the implementation of the theoretical background necessary to construct the profile databases. 


\section{Conclusion}

This work has demonstrated the proof-of-concept that orientation maps can be obtained by other means than EBSD and X-Ray diffraction. The new method presented here relies on the acquisition of a series of ion-induced SE images in a FIB, presenting a contrast due to the channeling effect. No additional hardware is necessary for this method. The first results, obtained on a TiN sample, show that an orientation map with a mean orientation error of less than $5^{\circ}$ can be obtained, by comparison with an EBSD map acquired on the same area, and considered as a reference. Preliminary tests have shown that only a few tens of images of the same ROI are necessary to compute an orientation map. The key idea of our method is the ability to compute a database of theoretical intensity profiles for any orientations. An experimental intensity profile extracted from the image series at a position $(\mathrm{X}, \mathrm{Y})$ is then compared to the database, with the best match giving the most probable orientation at this position.

Experimentally, a large number of microscope parameters have to be refined to determine the best acquisition conditions, in relation with the studied materials. For instance, the acceleration voltage, the detector type or the working distance were not varied during our experiments, therefore more work needs to be done to understand their role. Theoretically, our program to generate the intensity profile for each orientation is quite simple. Further experiments will be carried out to evaluate how this new method can be used (i) for non-cubic materials, (ii) for multi-phased materials and (iii) with an electron beam instead of an ion beam.

Aside from the possibility to obtain orientation maps, this work also provides two interesting ways to improve the characterization and understanding of microstructures by means of ion or electron microscopy. At first, acquiring a rotation series of images may significantly ease the detection of grain boundaries in polycrystals by simply averaging all the images as depicted in figure 8. Secondly, it has been shown that the crystallographic structure factor of the planes under the beam seems to drive their loss in intensity, when the beam arrives parallel to the corresponding 
plane. This is an alternative view that could complete the "particle" theory usually invoked to explain the channeling by ions [18].

Acknowledgments: the CLYM ( $\underline{w w w . c l y m} . f r)$ is gratefully acknowledged for the access to the FIB instrument which was financially supported by the CNRS, the Région Rhône-Alpes, the 'GrandLyon' and the French Ministry of Research and Higher Education.

\section{References}

[1] A.J. Schwartz, M. Kumar, B.L. Adams, D.P. Field, Electron backscatter diffraction in materials science, Springer, 2009.

[2] W. Yang, B. Adams, M. De Graef, Adaptive orientation imaging microscopy, in: (ICOTOM 12): 12 th International Conference on Textures of Materials, 1999, pp. 192-197.

[3] T. Maitland, A. Gholinia, Advances in High-Speed EBSD Orientation Mapping, Microscopy and Microanalysis, 13 (2007) 924-925.

[4] T. Britton, A. Wilkinson, Measurement of residual elastic strain and lattice rotations with high resolution electron backscatter diffraction, Ultramicroscopy, 111 (2011) 1395-1404.

[5] X. Tao, A. Eades, Errors, artifacts, and improvements in EBSD processing and mapping, Microscopy and Microanalysis, 11 (2005) 79-87.

[6] C. Maurice, R. Fortunier, A 3D Hough transform for indexing EBSD and Kossel patterns, Journal of microscopy, 230 (2008) 520-529.

[7] S.I. Wright, J. Zhao, B. Adams, Automated determination of lattice orientation from electron backscattered Kikuchi diffraction patterns, Texture, stress and microstructure, 13 (1991) 123-131.

[8] Y.-H. Chen, S.U. Park, D. Wei, G. Newstadt, M. Jackson, J.P. Simmons, M. De Graef, A.O. Hero, A Dictionary Approach to EBSD Indexing, arXiv preprint arXiv:1502.07436, (2015).

[9] E. Rauch, M. Véron, J. Portillo, D. Bultreys, Y. Maniette, S. Nicolopoulos, Automatic crystal orientation and phase mapping in TEM by precession diffraction, Microscopy and Analysis-UK, (2008) S5.

[10] E. Rauch, L. Dupuy, Rapid spot diffraction patterns idendification through template matching, Archives of Metallurgy and Materials, 50 (2005) 87-99.

[11] L. Reimer, Scanning electron microscopy: physics of image formation and microanalysis, Measurement Science and Technology, 11 (2000) 1826.

[12] S. Zaefferer, N.-N. Elhami, Theory and application of electron channelling contrast imaging under controlled diffraction conditions, Acta Materialia, 75 (2014) 20-50.

[13] M. Phaneuf, Applications of focused ion beam microscopy to materials science specimens, Micron, 30 (1999) 277-288. 
[14] Y. Yahiro, K. Kaneko, T. Fujita, W.-J. Moon, Z. Horita, Crystallographic orientation contrast associated with $\mathrm{Ga}+$ ion channelling for $\mathrm{Fe}$ and $\mathrm{Cu}$ in focused ion beam method, Journal of Electron Microscopy, 53 (2004) 571-576.

[15] J.R. Silk, R.J. Dashwood, R.J. Chater, Determination of lattice orientation in aluminium alloy grains by low energy gallium ion-channelling, Nuclear Instruments and Methods in Physics Research Section B: Beam Interactions with Materials and Atoms, 268 (2010) 2064-2068.

[16] V. Veligura, G. Hlawacek, R. van Gastel, H.J. Zandvliet, B. Poelsema, Channeling in helium ion microscopy: Mapping of crystal orientation, Beilstein journal of nanotechnology, 3 (2012) 501-506.

[17] L. Giannuzzi, J. Michael, Comparison of channeling contrast between ion and electron images, Microscopy and Microanalysis, 19 (2013) 344-349.

[18] R. Levi-Setti, T.R. Fox, K. Lam, Ion channeling effects in scanning ion microscopy with a $60 \mathrm{keV}$ Ga< sup $>+</$ sup > probe, Nuclear instruments and methods in Physics Research, 205 (1983) 299-309.

[19] R. Franklin, E. Kirk, J. Cleaver, H. Ahmed, Channelling ion image contrast and sputtering in gold specimens observed in a high-resolution scanning ion microscope, Journal of materials science letters, 7 (1988) 39-41.

[20] J. Guyon, H. Mansour, N. Gey, M. Crimp, S. Chalal, N. Maloufi, Sub-micron resolution selected area electron channeling patterns, Ultramicroscopy, 149 (2015) 34-44.

[21] J. Orloff, L. Swanson, M. Utlaut, Fundamental limits to imaging resolution for focused ion beams, Journal of Vacuum Science \& Technology B, 14 (1996) 3759-3763.

[22] J. Schindelin, I. Arganda-Carreras, E. Frise, V. Kaynig, M. Longair, T. Pietzsch, S. Preibisch, C. Rueden, S. Saalfeld, B. Schmid, Fiji: an open-source platform for biological-image analysis, Nature methods, 9 (2012) 676-682.

[23] P. Thevenaz, U.E. Ruttimann, M. Unser, A pyramid approach to subpixel registration based on intensity, Image Processing, IEEE Transactions on, 7 (1998) 27-41.

[24] R. Elmasri, S. Navathe, Fundamentals of Database Systems sixth Edition Pearson Education, (2011).

[25] A. Guttman, R-trees: a dynamic index structure for spatial searching, ACM, 1984.

[26] N. Roussopoulos, S. Kelley, F. Vincent, Nearest neighbor queries, in: ACM sigmod record, ACM, 1995, pp. 71-79.

[27] V. Randle, O. Engler, Introduction to texture analysis: macrotexture, microtexture and orientation mapping, CRC Press, 2000.

[28] H. Wenk, U. Kocks, The representation of orientation distributions, Metallurgical Transactions A, 18 (1991) 1083-1092.

[29] A. Day, T. Quested, A comparison of grain imaging and measurement using horizontal orientation and colour orientation contrast imaging, electron backscatter pattern and optical methods, Journal of microscopy, 195 (1999) 186-196.

[30] S.I. Wright, M.M. Nowell, R. de Kloe, P. Camus, T. Rampton, Electron imaging with an EBSD detector, Ultramicroscopy, 148 (2015) 132-145.

[31] C. Wu, B. Adams, C. Bauer, D. Casasent, A. Morawiec, S. Ozdemir, A. Talukder, Mapping the mesoscale interface structure in polycrystalline materials, MICROSCOPY AND MICROANALYSIS-NEW YORK-, 5 (1999) 260-261. 